\title{
The CARE guidelines: consensus-based clinical case reporting guideline development
}

\author{
Joel J Gagnier, ${ }^{1,2}$ Gunver Kienle, ${ }^{3}$ Douglas G Altman, ${ }^{4}$ David Moher, ${ }^{5}$ \\ Harold Sox ${ }^{6}$ David Riley, ${ }^{7}$ the CARE Group
}

\section{ABSTRACT}

A case report is a narrative that describes, for medical, scientific or educational purposes, a medical problem experienced by one or more patients. Case reports written without guidance from reporting standards are insufficiently rigorous to guide clinical practice or to inform clinical study design. Develop, disseminate and implement systematic reporting guidelines for case reports. We used a three-phase consensus process consisting of (1) premeeting literature review and interviews to generate items for the reporting guidelines, (2) a face-to-face consensus meeting to draft the reporting guidelines and (3) postmeeting feedback, review and pilot testing, followed by finalisation of the case report guidelines. This consensus process involved 27 participants and resulted in a 13-item checklist-a reporting guideline for case reports. The primary items of the checklist are title, key words, abstract, introduction, patient information, clinical findings, timeline, diagnostic assessment, therapeutic interventions, follow-up and outcomes, discussion, patient perspective and informed consent. We believe the implementation of the CARE (CAse REport) guidelines by medical journals will improve the completeness and transparency of published case reports and that the systematic aggregation of information from case reports will inform clinical study design, provide early signals of effectiveness and harms, and improve healthcare delivery.

\footnotetext{
${ }^{1}$ Department of Orthopaedic Surgery, University of Michigan, Ann Arbor, Michigan, USA; ' 2 Department of Epidemiology, School of Public Health, University of Michigan, Ann Arbor, Michigan, USA; ${ }^{3}$ Institute for Applied Epistemology and Medical Methodology, University of Witten/Herdecke, Freiburg, Germany; ${ }^{4}$ Centre for Statistics in Medicine, University of Oxford, Oxford, UK; ${ }^{5}$ Department of Epidemiology and Community Medicine, Ottawa Hospital Research Institute, Ottawa, Canada, University of Ottawa, Ottawa, Ontario, Canada; ${ }^{6}$ The Dartmouth Institute and Geisel School of Medicine at Dartmouth, Hanover, New Hampshire, USA; ${ }^{7}$ Global Advances in Health and Medicine, Portland, Oregon, USA
}

Correspondence to David Riley, driley@gahmllc.com

\section{INTRODUCTION}

A case report is a detailed narrative that describes, for medical, scientific, or educational purposes, a medical problem experienced by one or several patients.

Case reports present clinical observations customarily collected in healthcare delivery settings. They have proved helpful in the identification of adverse and beneficial effects, the recognition of new diseases, unusual forms of common diseases and the presentation of rare diseases. ${ }^{1}$ For example, our understanding of the relationship between thalidomide and congenital abnormalities ${ }^{2}$ and the use of propranolol for the treatment of infantile haemangiomas began with case reports. ${ }^{3}$ Case reports may generate hypotheses for future clinical studies, prove useful in the evaluation of global convergences of systems-oriented approaches and guide the individualisation and personalisation of treatments in clinical practice. ${ }^{4} 5$ Furthermore, case reports offer a structure for case-based learning in healthcare education and may facilitate the comparison of healthcare education and delivery across cultures.

Case reports are common and account for a growing number of articles in medical journals ${ }^{6}$; however, their quality is uneven. ${ }^{78}$ For example, one study evaluated 1316 case reports from four peerreviewed emergency-medicine journals and found that more than half failed to provide information related to the primary treatment that would have increased transparency and replication. ${ }^{9}$ Written without the benefit of reporting guidelines, case reports often are insufficiently rigorous to be aggregated for data analysis, inform research design or guide clinical practice. $^{79}$

Reporting guidelines exist for a variety of study designs including randomised controlled trials (Consolidated Standards of Reporting Trials, CONSORT), ${ }^{10}$ observational studies (Strengthening the Reporting of Observational studies in Epidemiology, STROBE) $^{11}$ and systematic reviews and meta-analyses (Preferred Reporting Items for Systematic Reviews and Meta-Analyses, PRISMA). ${ }^{12}$ Empirical evidence suggests that a journal's adoption of the CONSORT statement as a guide to authors is associated with an increase in the completeness of published randomised trials. ${ }^{13}$ Guidelines have been developed for adverse-event case reports ${ }^{14}$; however, general reporting guidelines for case reports do not exist. Our primary objective was to develop reporting guidelines for case reports through a consensus-based process.

\section{METHODS}

\section{Research design}

We followed the Guidance for Developers of Health Research Reporting Guidelines $^{15}$ and developed a three-phase consensus process. ${ }^{16}$ This consisted of (1) a premeeting literature review followed by interviews to generate items for a case report checklist, (2) a face-to-face consensus meeting for drafting a reporting guideline and (3) postmeeting feedback and pilot testing followed by finalisation of the case report guidelines.

\section{Participants}

We contacted 28 individuals who fulfilled at least one of the four criteria ${ }^{17-19}$ (1) publication of articles related to case reports; (2) publication of a manual, handbook or method guidelines related to case reports; (3) publication of a systematic review of methods or reporting related to case reports and (4) publication of other reporting guidelines for clinical research.

\section{Consensus process}

Phase I: Four of the authors, the steering committee (JG, GK, DM and DR), searched the literature for publications on the role of case reports, recommendations for their publication and surveys on reporting quality. A letter was sent to 28 potential participants explaining the purpose of the meeting, details of the consensus technique, and requesting their participation in generating specific recommendations for case reporting. Twenty-seven people agreed to participate and were scheduled for a telephone interview and sent a selection of key articles on case reports. During the telephone interview, participants were asked (1) what information was required to be included in case-reporting guidelines, (2) the rationale for their suggestions and (3) for references that supported their reasoning.

Three of the authors (JG, GK and DR) grouped the recommendations from the literature search and interviews by theme 
together with their rationale, references and operational definitions. No quantitative scoring was performed.

Phase II: The face-to-face consensus meeting at the University of Michigan in Ann Arbor (October 2012) included 18 participants from phase I, one research assistant and two student observers. The meeting began with a review of the blinded recommendations elicited during the phase I interviews, in whole group and small group sessions. On the second day, open discussion of each potential item continued, during which clarifications, opinions, justifications, operational definitions and new ideas were expressed. By the end of the second day, the group had agreed upon a set of preliminary reporting recommendations.

Phase III: The draft checklist was refined by the steering committee and sent for two rounds of review to the complete group (phases I and II participants). The finalised reporting guidelines incorporated the feedback from the entire CARE group.

\section{RESULTS}

The CAse REport (CARE) guidelines checklist is structured to correspond with key components of a case report and capture useful clinical information (including 'meaningful use' information mandated by some insurance plans).

The checklist begins with a statement that describes the narrative of a case report. The meeting CARE group felt that a case report should tell a story using prose that has a consistent style across all sections, including the rationale for any conclusions and take-away messages.

We recommend a timeline (item 7) in the form of a table or figure that gives the specific dates and times of important components of the case. This might include family and medical history, genetic information, current symptoms, diagnostic test results, interventions and events that occurred during follow-up. The timeline should show how the key events of the case unfolded.

We created separate checklist items for diagnostic assessments (item 8) and therapeutic interventions (item 9) with the recognition that both items will often be relevant in a case report.

The group discussed at length whether to include the patient's perspective on his or her experience. In the end, we advocated for patient-reported outcomes and experiences whenever possible (item 12). There was also discussion about the need for guidelines for patient-reported outcomes of their care. In a similar vein, a recent extension of the CONSORT statement was published for patient-reported outcomes in randomised trials; CONSORT-PRO. ${ }^{20}$

Finally, we included an item on informed consent (item 13). We believe that authors have an ethical duty to obtain informed consent from the patient to publish patient information in a case report. Consent becomes informed when the patient or a relative reads the case report and approves its contents. If the patient cannot give consent and attempts to find a relative to give proxy consent have failed, the authors should seek permission to publish from an institutional committee. There may be other circumstances where an ethics committee or Institutional Review Board (IRB) approval may be necessary. The CARE guidelines are shown in the following table 1 .

\section{DISCUSSION}

This 13-item checklist provides a framework to satisfy the need for completeness and transparency for published case reports. We attempted to strike a balance between adequate detail and the concise writing that is one of the appealing characteristics of a case report. Our consensus process resulted in a set of essential items for authors to consider when submitting a case report for publication.

While case reports have long been an important source of new ideas and information in medicine, ${ }^{21}$ it appears that case reports are likely to begin to play a role in the discovery of what works and for whom. BioMed Central launched the Journal of Medical Case Reports in $2007^{22}$ and a Cases Database in 2012 with more than 11000 published case reports from 50 medical journals. In 6 months, it has grown to more than 26000 case reports from 212 medical journals. ${ }^{23}$ The CARE guidelines checklist is part of a growing effort to improve the reporting of case reports.

There is substantial empirical evidence that reporting guidelines improve the completeness of published scientific reports. ${ }^{13} 2425$ A recent Cochrane review examining the influence of journal endorsement of the CONSORT statement on reporting included 53 publications assessing 16604 randomised controlled trials and found that CONSORT-endorsing journals consistently have better overall reporting. ${ }^{13}$ However, the potential impact of the CONSORT statement and related reporting guidelines has not been fully realised. A study examining the instructions to peer reviewers of 116 health research journals found that only $41(35 \%)$ provided online instructions to peer reviewers. Of those, only $19(46 \%)$ mentioned or referred to reporting guidelines as a useful resource. $^{26}$ In response, the authors provide several recommendations for editors to improve the peer review of submitted manuscripts, suggesting that journals have a responsibility to support peer reviewers. $^{26}$

The developers of reporting guidelines have a responsibility to plan a dissemination and implementation strategy that supports guidelines utilisation. ${ }^{15}$ Our efforts have several components:

- The CARE guidelines will be presented at international conferences and workshops including the Peer Review and Biomedical Publication Congress in Chicago on 10 September 2013.

- This article will be published simultaneously in multiple medical journals and outreach to the 212 journals depositing case reports into the BioMed Central Case Report Database.

- We will develop a more detailed explanation and elaboration article to outline the rationale for each item and include empirical evidence and examples of good reporting from published case reports.

- The CARE guidelines are being pilot tested, and preliminary results support the guidelines as currently written (personal communication with Helmut Kiene, Erica Oberg, Bill Manahan). Guidelines extensions for specialties are being developed.

- The CARE guidelines and related documents will be available on a dedicated website (www.CARE-statement. org), the EQUATOR Network website (www.equator-network.org) and translated into multiple languages.

- Authors, journal editors, peer reviewers and the wider medical community are encouraged to use the CARE checklist and provide feedback that can be incorporated into regular updates of the CARE guidelines.

- We will conduct and support research into the impact of the CARE guidelines on the reporting of case reports.

\section{LIMITATIONS}

The CARE guidelines and their development have several possible limitations. First, these guidelines were developed through a consensus method and thus represent the opinions of the participants. However, consensus was easily reached during our meeting, we referred to the empirical evidence where available, and we received feedback from a wide selection of individuals, beyond those involved 
Table 1 The CARE guidelines checklist

The narrative: A case report tells a story in a narrative format that includes the presenting concerns, clinical findings, diagnoses, interventions, outcomes (including adverse events) and follow-up. The narrative should include a discussion of the rationale for any conclusions and any take-away messages.

\begin{tabular}{|c|c|c|}
\hline Item name & $\begin{array}{l}\text { Item } \\
\text { no. }\end{array}$ & Brief description \\
\hline Title & 1 & $\begin{array}{l}\text { The words 'case report' (or 'case study') should appear in the title along with phenomenon of greatest interest (eg, symptom, diagnosis, } \\
\text { test, intervention) }\end{array}$ \\
\hline Keywords & 2 & The key elements of this case in $2-5$ words \\
\hline Abstract & 3 & $\begin{array}{l}\text { a) Introduction-What does this case add? } \\
\text { b) Case Presentation: } \\
\text { - The main symptoms of the patient } \\
\text { - The main clinical findings } \\
\text { - The main diagnoses and interventions } \\
\text { - The main outcomes } \\
\text { c) Conclusion-What were the main 'take-away' lessons from this case? }\end{array}$ \\
\hline Introduction & 4 & Brief background summary of this case referencing the relevant medical literature \\
\hline Patient information & 5 & $\begin{array}{l}\text { a) Demographic information (eg, age, gender, ethnicity, occupation) } \\
\text { b) Main symptoms of the patient (his or her chief symptoms) } \\
\text { c) Medical, family, and psychosocial history-including diet, lifestyle, and genetic information whenever possible, and details about } \\
\text { relevant comorbidities including past interventions and their outcomes }\end{array}$ \\
\hline Clinical findings & 6 & Describe the relevant physical examination (PE) findings \\
\hline Timeline & 7 & Depict important dates and times in this case (table or figure). \\
\hline $\begin{array}{l}\text { Diagnostic } \\
\text { assessment }\end{array}$ & 8 & $\begin{array}{l}\text { a) Diagnostic methods (eg, PE, laboratory testing, imaging, questionnaires) } \\
\text { b) Diagnostic challenges (eg, financial, language/cultural) } \\
\text { c) Diagnostic reasoning including other diagnoses considered } \\
\text { d) Prognostic characteristics (eg, staging) where applicable }\end{array}$ \\
\hline $\begin{array}{l}\text { Therapeutic } \\
\text { intervention }\end{array}$ & 9 & $\begin{array}{l}\text { a) Types of intervention (eg, pharmacologic, surgical, preventive, self-care) } \\
\text { - Administration of intervention (eg, dosage, strength, duration) } \\
\text { - Changes in intervention (with rationale) }\end{array}$ \\
\hline $\begin{array}{l}\text { Follow-up and } \\
\text { outcomes }\end{array}$ & 10 & $\begin{array}{l}\text { a) Summarise the clinical course of all follow-up visits including } \\
\text { - Clinician and patient-assessed outcomes } \\
\text { - Important follow-up test results (positive or negative) } \\
\text { - Intervention adherence and tolerability (and how this was assessed) } \\
\text { - Adverse and unanticipated events }\end{array}$ \\
\hline Discussion & 11 & $\begin{array}{l}\text { a) The strengths and limitations of the management of this case } \\
\text { b) The relevant medical literature } \\
\text { c) The rationale for conclusions (including assessments of cause and effect) } \\
\text { d) The main 'take-away' lessons of this case report }\end{array}$ \\
\hline Patient perspective & 12 & The patient should share his or her perspective or experience whenever possible \\
\hline Informed consent & 13 & Did the patient give informed consent? Please provide if requested \\
\hline
\end{tabular}

in our consensus meeting. Second, we recognise that causality determinations are a challenge for case reports even when following reporting guidelines. ${ }^{27} 28$ The CARE guidelines emphasise information quality independent of causality assessments. Different specialties, practitioners, and patients are likely to require extensions of the CARE guidelines with specialty specific information. We welcome discussions with groups interested in using the CARE guidelines as the basis for their specific reporting needs.

Though not mentioned in our guidelines, medical journals often require authors to address three issues: (1) potential competing interests, (2) de-identification of patientrelated data and (3) ethics committee or IRB approval if obtained or necessary.

\section{CONCLUSIONS}

Anticipating a long future for case reports, we have provided guidance in the form of reporting standards for use by healthcare stakeholders around the world. The growth of case reports in an era in which clinical trials and systematic reviews dominate the tables of content of medical journals indicates that case reports have value, particularly with the increasing importance of individualised care. Unlike randomised controlled trials, case reports are individual reports related to the care of individual patients where the sample size is one. When systematically collected and combined into larger datasets, they can be analysed, enhancing the early discovery of effectiveness and harms.

We anticipate that the analysis of systematically aggregated information from patient encounters (now mandated by some insurance plans) will provide scalable, data-driven insights into what works for which patients in real time, facilitating comparisons across medical systems and cultures. Practitioners will soon be able to provide-and in some cases they are required to provide-patients with information from their encounters. This will transform how we think about 'evidence' and revolutionise its creation, diffusion and use-opening new opportunity landscapes. When it becomes clear how new data contributes to evidence, the stewardship needed to produce high-quality data will be more rewarding and our attitude towards 'observation' will shift. The CARE guidelines provide a framework to satisfy the need for precision, completeness and transparency.

Authors note Joel J Gagnier, University of Michigan, and David Riley, Global Advances in Health and Medicine, organised this consensus-based guideline-development project. The volunteer steering committee consisted of Joel J Gagnier, Gunver Kienle, David Moher, and David Riley.

Collaborators The CARE group: Alyshia Allaire, BS, Portland, OR, USA, Douglas G Altman, DSc, Centre for Statistics in Medicine, University of Oxford, Oxford, UK, 
Jeffrey Aronson, MB, ChB, MA Dphil, FRCP, FB, PharmacolS, University of Oxford, Oxford, UK*, James Carpenter, MD, Department of Orthopaedic Surgery, University of Michigan, Ann Arbor, MI, USA, Joel J Gagnier, ND, MSc, PhD, Departments of Orthopaedic Surgery and Epidemiology, University of Michigan, Ann Arbor, MI, USA, Patrick Hanaway, MD, Director of Medical Education, Institute for Functional Medicine Asheville, NC, USA* , Carolyn Hayes, PhD, RN, NEA-BC, Dana-Farber Brigham and Women's Cancer Center, Boston, MA, USA, David Jones, MD, President, Institute for Functional Medicine, Ashland, OR, USA, Marietta Kaszkin-Bettag, PhD, University of Frankfurt, Pharmalex GmbH, Mannheim, Germany, Michael Kidd, AM, Editor-in-Chief Journal of Medical Case Reports, Faculty of Health Sciences, Flinders University, Adelaide, Australia*, Helmut Kiene, Dr med, Editor, Global Advances in Health and Medicine, Institute for Applied Epistemology and Research Methodology, University of Witten/Herdecke, Freiburg, Germany, Gunver Kienle, Dr med, Editor, Global Advances in Health and Medicine, Institute for Applied Epistemology and Research Methodology, University of Witten/Herdecke, Freiburg, Germany, Ben Kligler, MD, MPH, Co-Editor-in-Chief Explore, Beth Israel Medicine Center, New York, NY USA*, Lori Knutson, RN, BSN, HN-BC, Integrative Healthcare Solutions, Minneapolis, MN, USA, Christian Koch, Dr med. Habil., PhD, FACP, FACE, Deputy Editor Journal of Medical Case Reports, University of Mississippi, Jackson, MS, USA*, Karen Milgate, MPP, Independent Health Policy Consultant, Washington, DC, USA*, Michele Mittelman, RN, MPH, Editor, Global Advances in Health and Medicine, Dover, MA, USA, David Moher, PhD, Ottawa Hospital Research Institute; Department of Epidemiology and Community Medicine, University of Ottawa, Ottawa, ON Canada*, Hanna Oltean, MPH, University of Michigan, Ann Arbor, Ml, USA, Greg Plotnikoff, MD, MTS, FACP, Editor, Global Advances in Health and Medicine, Allina Center for Healthcare Innovations and the Penny George Institute for Health and Healing, Minneapolis, MN, USA, Richard Alan Rison, MD, FAANEM, Deputy Editor, Journal of Medical Case Reports, Section Editor, BMC Research Notes, PIH Health Hospital, Whittier, University of Southern California, Los Angeles, CA, USA*, David Riley MD, Editor-in-Chief, Global Advances in Health and Medicine, Portland, OR, USA, Anil Sethi, MS, Johns Hopkins School of Medicine-Information architecture and IT, Palo Alto, CA, USA* Larissa Shamseer, MSc, Ottawa Hospital Research Institute, Ottawa, ON, Canada, Richard Smith, MB, ChB, MSc, United Healthcare Chronic Disease Initiative, London, UK, Harold Sox, MD, The Dartmouth Institute and Geisel School of Medicine at Dartmouth, Hanover, $\mathrm{NH}_{\text {, }}$ USA, Peter Tugwell, MD, FRCP, University of Ottawa, Ottawa, ON, Canada. * Participated in the guidelines development process, the review and editing of the CARE guidelines and this article, but did not attend the face-to-face consensus meeting.

Contributors JJG and DR wrote the first draft of the article. DGA, JJG, GK, DM, DR and HS critically reviewed and edited drafts. The entire CARE group participated in parts or all of the guidelines development process and contributed to the editing and revision of the CARE guidelines and this article.
Funding The Department of Orthopaedic Surgery, the Office of the Vice-President of Research at the University of Michigan, and Global Advances in Health and Medicine provided funding for this project. David Moher is funded through a University of Ottawa Research Chair. Funding support was used to reimburse the travel-related expenses of conference attendees. There were no honoraria.

\section{Competing interests None.}

Provenance and peer review Not commissioned; internally peer reviewed.

Open Access This is an Open Access article distributed in accordance with the Creative Commons Attribution Non Commercial Non Derivative (CC BY-NCND 3.0) license, which permits others to copy, distribute and transmit the work, provided the original work is properly cited and the use is noncommercial. See: http://creativecommons.org/licenses/by-nc-nd/3.0

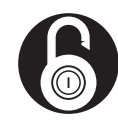

\section{OPEN ACCESS}

To cite Gagnier JJ, Kienle G, Altman DG, et al. Global Adv Health Med Published online: [please include Day Month Year] doi:10.1136/bcr-2013-201554

Received 28 August 2013

Accepted 5 September 2013

Reproduced with permission from Global Advances in Health and Medicine.

\section{REFERENCES}

1 Hauben M, Aronson JK. Gold standards in pharmaco-vigilance: the use of definitive anecdotal reports of adverse drug reactions as pure gold and high-grade ore. Drug Saf 2007;30:645-55.

2 Vandenbroucke JP. Thalidomide: an unanticipated adverse effect. JLL Bulletin: commentaries on the history of treatment evaluation. http://www. jameslindlibrary.org/illustrating/articles/ thalidomide-an-unanticipated-adverse-effect (accessed 10 Jan 2013)

3 Levy M. Propranolol for infantile hemangiomas. Global Adv Health Med 2012;1:14-16.

4 Jenicek M. Clinical case reporting in evidence-based medicine. 2nd edn. New York: Oxford University Press, 2001.

5 Riley D. Case reports in the era of clinical trials. Global Adv Health Med 2013;2:10-11.

6 "Case reports" search results. Bethesda, MD: PubMed, US National Library of Medicine, 2013 http://www.ncbi.nlm.nih.gov/pubmed/?term=case +reports (accessed 10 Jun 2013).

7 Kaszkin-Bettag M, Hildebrandt W. Case report on cancer therapies: the urgent need to improve the reporting quality. Global Adv Health Med 2012;1:8-10.

8 Kljakovic M. Single cases in general practice and general medical journals. Aust Fam Physician 2002;31:669-73.
9 Richason TP, Paulson SM, Lowenstein SR, et al. Case reports describing treatments in the emergency medicine literature: missing and misleading information. BMC Emerg Med 2009;9:10.

10 Schulz KF, Altman DG, Moher D. CONSORT 2010 statement: updated guidelines for reporting parallel group randomized trials. Ann Intern Med 2010;152:726-32.

11 von Elm E, Altman DG, Egger $M$, et al. TROBE Initiative. The strengthening the reporting of observational studies in epidemiology (STROBE) statement: guidelines for reporting observational studies. BMJ 2007;335:806-8.

12 Moher D, Liberati A, Tetzlaff J, et al, the PRISMA Group. Preferred reporting items for systematic reviews and meta-analyses: the PRISMA Statement. PLoS Med 2009;6:e1000097.

13 Turner L, Shamseer L, Altman DG, et al. Does use of the CONSORT statement impact the completeness of reporting of randomised controlled trials published in medical journals? A Cochrane review. Syst Rev 2012;1:60.

14 Kelly WN, Arellano FM, Barnes J, et al. Guidelines for submitting adverse event reports for publication. Drug Saf 2007;30:367-73.

15 Moher D, Schulz KF, Simera I, et al. Guidance for developers of health research reporting guidelines. PLoS Med 2010;7:e1000217.

16 Williams PL, Webb C. The Delphi technique: a methodological discussion. J Adv Nurs 1994;19: 180-6.

17 Thomas B. Using nominal group technique to identify researchable problems. J Nurs Educ 1983;22:335-7.

18 Tully MP, Cantrill JA. Exploring the domains of appropriateness of drug therapy using the nominal group technique. Pharm World Sci 2002;24:128-31.

19 Horton JN. Nominal group technique: a method of decision-making by committee. Anaesthesia 1980:35:11-14.

20 Calvert M, Blazeby J, Altman DG, et al. CONSORT PRO Group. Reporting of patient-reported outcomes in randomized trials: the CONSORT PRO extension. JAMA 2013:309:814-22.

21 Vandenbroucke JP. In defense of case reports and case series. Ann Intern Med. 2001;134:330-4.

22 Journal of Medical Case Reports. http://www. jmedicalcasereports.com/ (accessed 17 Jun 2013).

23 Cases Database. http://www.casesdatabase.com/ (accessed 17 Jun 2013)

24 Hopewell S, Clarke M, Moher D, et al. CONSORT for reporting randomized controlled trials in journal and conference abstracts: explanation and elaboration. PLoS Med 2008;5:e20.

25 Hopewell S, Ravaud P, Baron G, et al. Effect of editors implementation of CONSORT guidelines on the reporting of abstracts in high impact medical journals: interrupted time series analysis. BMJ 2012:344:e4178.

26 Hirst A, Altman DG. Are peer reviewers encouraged to use reporting guidelines? A survey of 116 health research journals. PLOS ONE 2012;7:e35621.

27 Hill $A B$. The environment and disease: association or causation? Proc $R$ Soc Med 1965:58:29-5.

28 Kiene $H$, Hamre $H$, Kienle $G$. In support of clinical case reports: a system of causality assessment. Global Adv Health Med 2013;2:76-87. 